\title{
The Epidemiology of Intentional Non-Fatal Self-Harm Poisoning in the United States: 2001-2004
}

\author{
Jane M. Prosser, $M D^{a}$, Jeanmarie Perrone, $M D^{b}$, Jesse M. Pines, $M D, M B A^{b}$ \\ aDepartment of Emergency Medicine, Temple University Hospital, Philadelphia, PA \\ bDepartment of Emergency Medicine, Hospital of the University of Pennsylvania, Philadelphia, PA
}

\begin{abstract}
Introduction: Suicide attempts through intentional poisoning are an important public health concern. This investigation sought to determine the different US incident rates of poisonings (with intent to do self-harm) by race, sex, and gender.

Methods: We used data from the National Electronic Injury Surveillance System - All Injury Program (NEISS-AIP) to describe differences in incidence rates of non-fatal, self-harm poisonings evaluated in US emergency departments (ED) from 2001 to 2004. WISQARS defines self-harm as "either confirmed or suspected from injury or poisoning resulting from a deliberate act inflicted on oneself with the intent to take one's own life or harm oneself."

Results: From 2001 to 2004, there were 976,974 (95\% Confidence Interval (CI) 772,884 - 1,181,065) non-fatal self-harm poisonings reported in US Emergency Departments. Young white females between 15 to 19 years of age were at highest risk with an incidence rate of 248 per 100,000 (95\% CI 180 - 315). Females had higher rates of self-harm poisonings than males: the rate for females was 101 per 100,000 (95\% CI 81 - 123); the rate for males was 66 per 100,000 (95\% CI $51-81), \mathrm{p}<0.001$. Whites had slightly higher rates than blacks, and significantly higher rates than Hispanics: whites rates were 71 per 100,000 (95\% 51 - 91); black rates were 65 per 100,000 (95\% CI 35 - 96), and Hispanic rates were 23 per 100,000 (95\% 10 - 37). There was a significant increase in the incidence rate from 2002 to 2003 ( $\mathrm{p}<0.001$ ). Incident rates decreased for females and males older than 19 years of age ( $<0.001$ for males and females).

Conclusion: Recently, the incidence of self-harm poisoning has risen considerably. It rose from 75 (CI 53-98) per 100,000 in 2002 to 94 (CI 74-115) per 100,000 in 2003; incidence remained relatively steady in 2004. Whites have higher incidence rates than blacks and significantly higher incidence rates than Hispanics. Females are at higher risk than males, and adolescent white females are at highest risk. An understanding of the demographic factors associated with self-harm poisoning may provide useful information to improve prevention and treatment strategies.
\end{abstract}

\section{INTRODUCTION}

Self-harm poisoning (with an intent to commit suicide) is an important public health concern in the US. Recent evidence indicates that the number of cases of self-harm poisoning has increased over the past 10 years [1]. While suicidal poisoning accounts for only $8 \%$ of all reported poisonings, overall mortal- ity rates from self-harm poisoning are considerably higher. Selfharm poisoning accounted for more than half of the 1183 reported poisoning fatalities in 2004 [2]. There were 90 fatalities in the 13 to 19 year age range; this is the highest number ever reported [2].

Public health interventions aim to reduce the morbidity and mortality of self-harm poisoning by limiting the availability of

Keywords: poisoning, self-harm, demographics, emergency department, epidemiology

Notes: This data was presented as poster at the North American Congress of Clinical Toxicology Meeting in San Francisco, CA in October 2006.

There was no outside funding of any kind used for this study.

Corresponding Author: Jane Prosser, MD, Temple University Hospital, Department of Emergency Medicine, 3401 N. Broad Street, Philadelphia, PA 19104. Email: jprosser100@hotmail.com 
potentially toxic medicines to at-risk-populations and by formulating medications to minimize potential medication toxicity. For example, European data has shown that limiting acetaminophen package size has reduced the morbidity and mortality in acetaminophen ingestions $[3,4]$. However, more specific interventions aimed at high-risk groups may help to improve outcomes of selfharm poisoning.

US data on poisoning is available from the Toxic Exposure Surveillance System (TESS) of the American Association of Poison Control Centers, but the information is not specific to race and ethnicity. In 2000, the National Electronic Injury Surveillance System - All Injury Program (NEISS-AIP) began collecting data on poisoning injuries from US emergency departments (ED). However, not much data have been published on the comparative demographics of self-harm poisoning using this database. An understanding of the epidemiology of patients who are at highest risk for self-harm poisoning may aid in the development of specific intervention strategies.

This investigation sought to determine the different US incident rates of poisonings (with intent to do self-harm) by race, sex, and gender. We hypothesized that young white females would be at highest risk.

\section{METHODS}

We performed a retrospective data analysis of poisonings from hospital emergency departments (EDs) in the United States that participate in the NEISS-AIP. The NEISS-AIP collects injury data from 66 EDs throughout the United States (US). The hospitals are a representative sample of all US emergency departments. Hospitals provide data from about 500,000 injury-related ED cases yearly. Data from these cases are weighted by the inverse of the probability of selection to provide national estimates. The NEISS-AIP data is potentially more complete than TESS because it is abstracted from the charts of participating hospitals nationwide. In contrast, the TESS data relies on health care providers to call the Poison Control center to report poisonings. National estimates of the incident rate of poisonings were determined from information provided by the Center for Disease Control's Webbased Injury Statistics Query and Reporting System (WISQARS) [5]. WISQARS, a web-based system, allows access to NEISS-AIP data.

For this analysis, we used the 2001 to 2004 nonfatal injury datasets from the National Center for Injury Prevention and Control. Through WISQARS, we queried the NEISS-AIP database for non-fatal, self-harm and poisoning categories. Results were stratified by age, gender, and race. WISQARS defines self-harm as "either confirmed or suspected from injury or poisoning resulting from a deliberate act inflicted on oneself with the intent to take one's own life or harm oneself." Self-harm includes suicide attempts and other intentional self-harm. WISQARS defines poisoning as the "ingestion, inhalation, absorption through the skin, or injection of so much of a drug, toxin (biologic or non-biologic), or other chemical that a harmful effect results, such as drug overdoses". This does not include harmful effects from normal therapeutic drugs (such as adverse effects from therapeutic drugs). Incident rates were abstracted by trained coders using a national sample of injuries at NEISS-AIP hospitals and then calculated. For each record, coded data and a narrative was transmitted to the Consumer Product Safety Division where quality assurance experts assigned direct cause to each case. We determined population rates based on the Population Estimates Program of the U.S. Census Bureau under a collaborative arrangement with the National Center for Health Statistics [5].

We used Stata 9.0 (College Station, TX, 2005) to calculate confidence intervals and to perform tests for data trends. The institutional review board at the University of Pennsylvania reviewed the study protocol and deemed this study exempt from further review.

\section{RESULTS}

From 2001 to 2004, there were 976,974 (95\% Confidence Interval 772,884 - 1,181,065) nonfatal, self-harm poisonings evaluated in EDs in US hospitals. Of those, 270,228 (95\% CI 208,494 $331,961)$ were treated and released, 199,666 were transferred to other facilities (95\% CI 129,480 - 269,852), 482,613 (95\% CI 352,916 - 612,309) were hospitalized, and 24,468 (95\% CI 13,038 35,898 ) were observed, left against medical advice, or had a disposition entered as unknown. In 2001, the yearly incidence rate was 76 (95\% CI 53 - 98) per 100,000, 75 (95\% CI 58 - 92) per 100,000 in 2002, 94 (95\% CI 74 - 115) per 100,000 in 2003, and 93 (95\% CI 74 - 112) per 100,000 in 2004. Incident rates significantly increased from 2002 to 2003 (p < 0.001). The relative increase in incidence was $23.7 \%$. Figure 1 shows the trend in incidence.

Females had higher rates of self-harm poisonings than males: females rates were 101 per 100,000 (95\% CI $81-123)$; males rates were 66 per 100,000 (95\% CI $51-81), \mathrm{p}<0.001$. Whites had slightly higher rates than blacks, and significantly higher rates than Hispanics: whites rates were 71 per 100,000 (95\% 51 - 91); black rates were 65 per 100,000 (95\% CI 35 - 96), and Hispanic rates were 23 per 100,000 (95\% 10 - 37). There was a significant increase in the rate of incidence from 2002 to 2003 ( $\mathrm{p}<0.001$ ). Incident rates decreased for females and males older than 19 years of age ( $p<0.001$ for males and females) (Figure 2). Young white females between 15 to 19 years of age are the demographic group at highest risk with an incidence rate of 248 per 100,000 (95\% CI $180-315)$.

\section{DISCUSSION}

Self-harm poisoning is a significant public health problem. The 2001 to 2004 data from the NIESS-AIP shows that the incidence of self-harm poisoning increased by nearly twenty-five percent, a significant increase over a three-year period. Although intentional self-poisoning is not uncommon, the data on epidemiologic risk factors (such as race or ethnicity) in cases of self-harm ingestion 


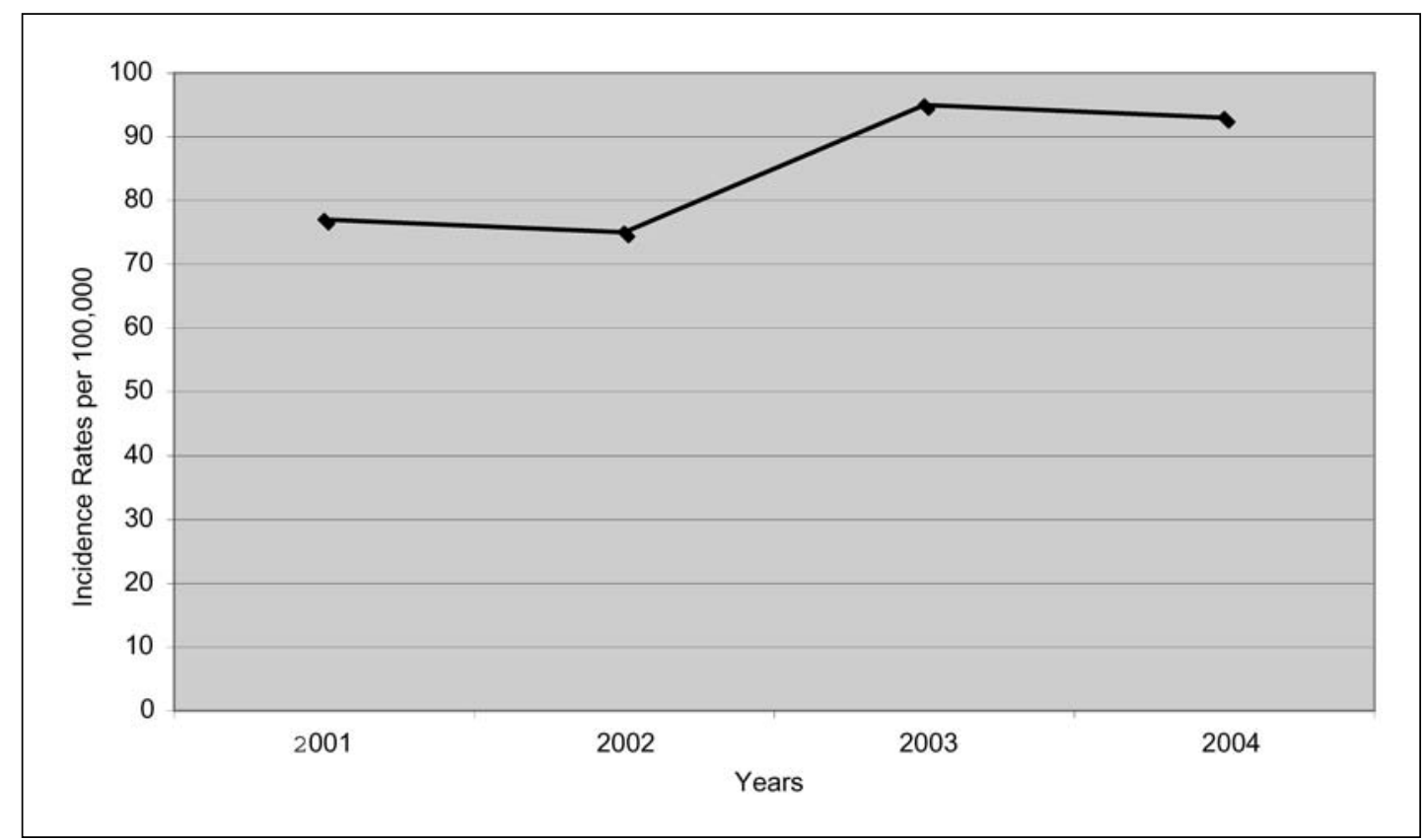

Figure 1: Increase in Incidence Rates of Intentional Nonfatal, Self-harm Poisonings per 100,000 using the NEISS-AIP Data from 2001-2004.

is lacking. The data regarding ethnicity in self-inflicted injury in general and in poisoning specifically is conflicting [6-13].

Our data differs from previous studies by exclusively examining nonfatal, self-harm poisoning. We did not review the incidence of fatal, self-harm poisoning in this study for several reasons. The epidemiology of fatal suicide poisoning has been extensively published in the literature, and we did not feel that adding this data to our study would enhance the previously existing body of literature. Also, fatal overdose is very underrepresented in hospital and poison center data because many victims of fatalities are taken directly to morgues or medical examiners' offices.

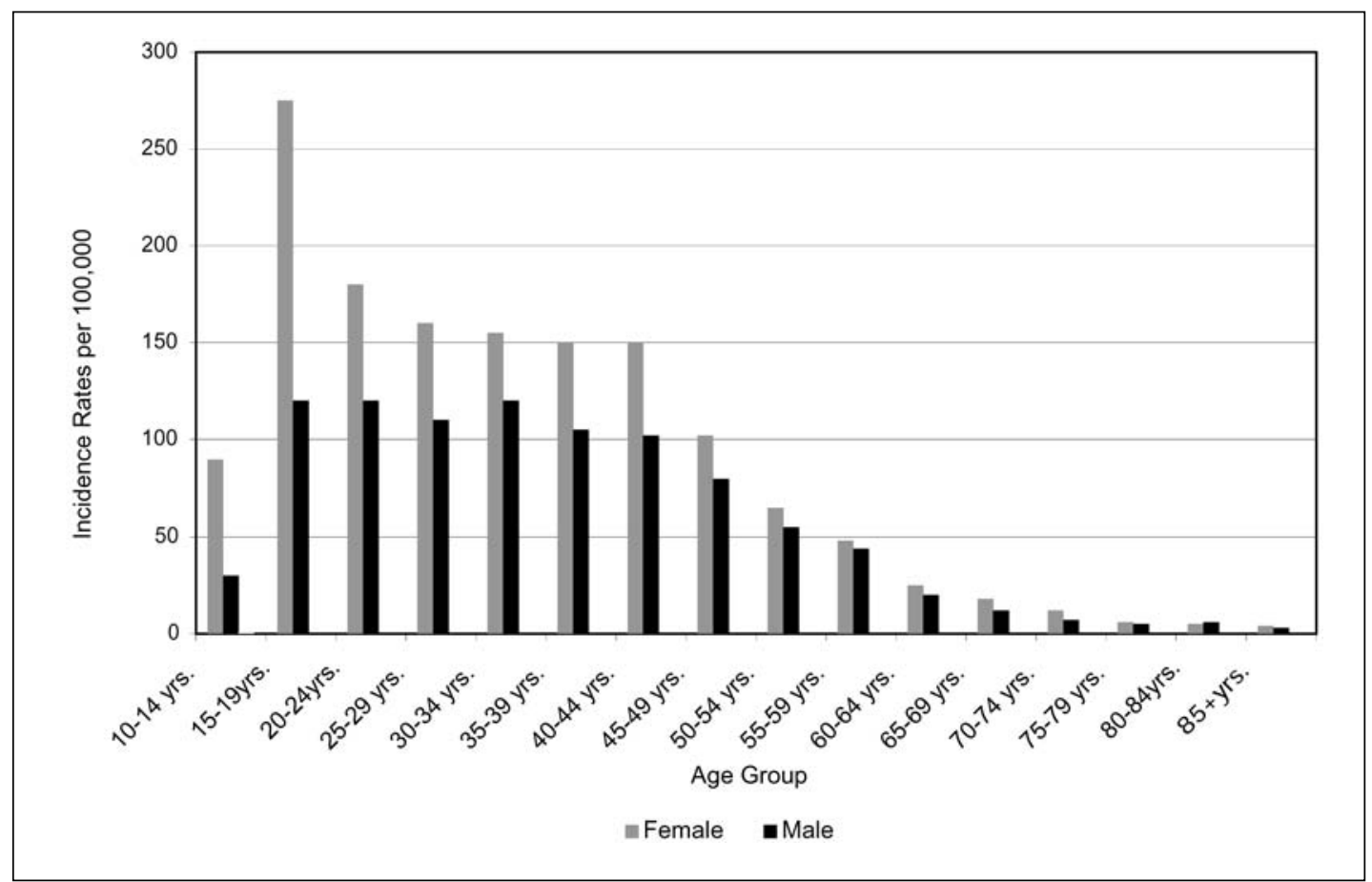

Figure 2: Incidence Rates for Intentional Nonfatal, Self-harm Poisonings Stratified by Age and Gender using NEISS-AIP Data from 2001-2004. 
Using the NEISS-AIP database, we were able determine national statistics relevant to the epidemiology of US selfpoisoning. It also allowed us to compile a much larger study population than local data would have permitted. We found intentional self-harm poisoning most common in adolescent, white females. This is consistent with our initial hypothesis. Rates were lower for black females and significantly lower for Hispanic females. Also, males had lower rates than females. White females 15 to 19 years of age had an incidence of intentional poisoning approximately 4 times higher than the general population.

Many studies about the ethnic differences in suicide attempts are surveys that ask participants to self-report prior suicide attempts. Data from the National Institute of Mental Health's Epidemiologic Catchment Area Study reported that whites have the highest rate of suicidal behavior [6]. However, the Youth Risk Behavior Survey found that Hispanics and blacks were significantly more likely than whites to attempt suicide [7]. These studies rely on patients to accurately self-report suicide attempts.

Shepard and Klein-Schwartz reviewed CDC data on adolescent poisoning deaths in the US from 1979-1994. They found that poisoning was the cause of suicide in $12.3 \%$ of deaths. In the 15-19 year age group, males were more likely to die from suicidal poisoning than females. However, females had a higher rate of suicide attempts by poisoning than males ( 89.5 per 100,000 vs. 36.6 per 100,000$)$. In adolescence 15 to 19 years of age, blacks' incidence of suicide was $70 \%$ lower than whites [8]. This study reviewed accidental and intentional poisoning deaths. The results were consistent with our data: females and whites show higher rates of poisoning suicide attempts.

Several studies have examined patients of suicide attempts who sought hospital care. In England, Merrill and Owens examined rates of ethnic differences in self-poisoning. They found the hospital admission rates of South Asian females nearly three times higher than that of white females. Rates for South Asian men were lower than white British men [9]. Spicer and Miller studied patients in eight US states and found slightly higher rates of attempted suicide among blacks [10]. Trinkoff et al described the characteristics of pediatric poisoning admissions to Maryland hospitals. In their study, white females had the highest rates of hospitalization and teenagers accounted for eighty percent of the mortality in pediatric overdoses [11]. The results of our study are similar to the findings in the Trinkoff study: white, adolescent females show the highest rates of self-harm poisoning.

Two recent papers examined the epidemiology of patients presenting to emergency departments with self-inflicted injuries. Ikeda et al (from the CDC) reported on the ethnicity of selfinflicted injuries in patients treated in emergency departments in 2000. They found that most self-inflicted injuries occurred in white adolescent females. Rates were also higher in white males than black males [12]. Doshi et al recently published epidemiologic data on patients with self-inflicted injuries who presented to US emergency departments. They found that $68 \%$ of patients who presented with self-inflicted injury chose self-poisoning as their method of injury. They also observed that suicide attempts occurred more commonly in adolescents and more commonly in females. However, they noted that blacks have a slightly higher rate of suicide attempts than whites [13]. These studies agree with our findings: self inflicted injury is more common in whites than blacks and is most common in white, adolescent females. However, neither of these studies specifically examined the characteristics of patients with self-harm poisonings. All of the datasets included firearms and other method of injury $[10,12,13]$.

The US Department of Health and Human Services initiative Healthy People 2010 aims to reduce the rate of suicide and reduce the rate of suicide attempts among adolescents. In order to reduce these rates, the initiative proposes "more targeted approaches [that] should consider risk factors most salient and appropriate for select populations" [14]. Public health interventions targeted at specific populations are beneficial in prevention of self-harm poisonings. The institution of a packet size limitation of acetaminophen in Europe has been correlated with the decreased morbidity and mortality from suicidal ingestions. For example, France limits acetaminophen packet size to 8 grams. Ganier et al. have reported that this intervention has resulted in a very low number of cases of hepatotoxicity and death from acetaminophen [3]. Similarly in England, reduction in the package size of over the counter analgesics such as acetaminophen was associated with a decrease in the number of suicidal overdoses [4].

\section{LIMITATIONS}

This study has several limitations. Sampling error is the major limitation. Nonsampling error in the data includes misclassification bias, nonresponse, and incomplete response errors. Additionally, the NIESS-AIP database uses record review-and so-International Classification of Diseases Ninth revision (ICD9) coding may miss or mischaracterize some poisoning cases. For example, cases may have been missed if the patient was not forthcoming about the self-poisoning (intentional or unintentional). Collected data only represents information gathered from ED reports; it does not include the additional data of patients admitted or transferred. This data represents an amalgamation of US ED visits for poisoning. Local rates of self-poisoning may vary and may not be accurately reflected in this study. This study reflects information derived from only one database that uses weighted numbers to derive national estimates.

\section{CONCLUSION}

The incidence of nonfatal, self-harm poisoning increased by almost 25\% between 2002 and 2003. The data presented in this investigation helps to clarify the demographics of self-harm poisoning. An understanding of which patients are at highest risk for self-harm poisoning attempts can aid in the development of prevention and treatment strategies. On an individual level, practitioners should consider the toxicity in overdose when prescribing mediations and treating patients with mental illness and substance abuse. When a patient in a high-risk group for intentional poisoning requires pharmacotherapy, practitioners should limit specific prescriptions in dose and number and avoid the most 
potentially lethal medications. Emergency department knowledge of the epidemiology of intentional poisoning with suicidal intent is important for the prescribing and disposition of patients at highest risk.

The authors have no potential conflicts of interest to report.

\section{REFERENCES}

1. Brickman, AL, Mintz, DC. Datapoints: U.S. rates of selfinflicted injuries and suicide, 1992-1999. Psychiatr Serv. 2003 Feb;54(2):168.2.

2. Watson WA, Litovitz TL, Rodgers Jr GC, Klein-Schwartz W, Reid N, Youniss J, Flanagan A, Wruk KM. 2004 annual report of the American Association of Poison Control Centers Toxic Exposure Surveillance System. Am J Emerg Med. 2005. 23(5):589-666

3. Ganier R, Bismuth C. Liver failure induced by paracetamol. BMJ 193;306:718.

4. Hawton K, Simkin S, Deeks J, Cooper J, Johnston A, Waters $\mathrm{K}$, et al. UK legislation on analgesic packs: before and after study of long term effect on poisonings. BMJ. 2004. 379:1076-1080

5. Centers for Disease Control and Prevention. Web-based Injury Statistics Query and Reporting System (WISQARS) [Online]. (2003). National Center for Injury Prevention and Control, Centers for Disease Control and Prevention (producer). Available from: URL: www.cdc.gov/ncipc/wisqars. [accessed May 23, 2006]
6. Moscicki EK, O'Carroll P, Rae DS, Locke BZ, Roy A, Regier DA. Suicide attempts in the epidemiologic catchment area study. Yale J Biol \& Med. 1988; 61:259-68.

7. Kann L, Warren CW, Harris WA Collins JL, Williams BI, Ross JG, et al. Youth risk behavior surveillance - United States, 1995. MMWR. 1996. 45(SS-4):1-86.

8. Shepard G, Klein-Schwartz W. Accidental and suicidal adolescent poisoning deaths in the United States, 1979-1994. Arch Pediatr Adolesc Med. 1998;152:1181-1185

9. Merrill J, Owens J. Ethnic differences in self-poisoning: a comparison of Asian and white groups. Br J Psychiatry.

1986; 148 :708-12.

10. Spicer RS, Miller TR. Suicide acts in 8 states: Incidence and case fatality rats by demographics and method. Am J Public Health. 2000;90(12):1885-1891

11. Trinkoff AM, Baker SP. Poisoning hospitalizations and deaths from solids and liquids among children and teenagers. Am J Public Health. 1986;76(6):657-660.

12. Ikeda R, Mahendra R, Saltzman L, Crosby A, Wills L, Mercy L, et al. Nonfatal self-inflicted injuries treated in hospital emergency departments: United States, 2000. MMWR Morb Mortal Wkly Rep. 2002;51:436-438.

13. Doshi A, Boudreaux ED, Wang N, Pelletier AJ, Camargo CA Jr. National study of US emergency department visits for attempted suicide and self-inflicted injury, 1997-2001. Ann Emerg Med. 2005;46:369-375.

14. Health People 2010 (2000) United States Department of Health and Human Services [Online]. Available from: URL: http://www.healthypeople.gov/Publications. [Last accessed 6/09/06] 